Facile manner in regulating the graphene-shelled structure of cobalt nanoparticles for the

\title{
synthesis of amines
}

Xiuzheng Zhuang ${ }^{1,2 \#}$, Jianguo Liu ${ }^{1,3^{*} \#, ~ L o n g l o n g ~} \mathrm{Ma}^{{ }^{1 *}}$

1 CAS Key Laboratory of Renewable Energy, Guangdong Provincial Key Laboratory of New and Renewable Energy Research and Development, Guangzhou Institute of Energy Conversion, Chinese Academy of Sciences, Guangzhou 510640, P. R. China.

2 University of Chinese Academy of Sciences, Beijing 100049, P. R. China.

3 Dalian National Laboratory for Clean Energy, Dalian 116023, P. R. China.

*Corresponding authors e-mail: liujg@ms.giec.ac.cn; mall@ms.giec.ac.cn

\# These authors contributed equally to this work.

Abstract: The synthesis of pharmaceutical relevant compounds keeps close ties with the development of metalbased catalysts, which remains a common goal in chemical research to solve challenges of application, such as the deactivation of catalyst caused by its tender structure or metal leaching. In this work, we reported a simple and manageable method for the preparation of heterogeneous catalyst with stable cobalt nanoparticles that encapsulated by the outer graphitic shell, whose catalytic activity and selectivity for the synthesis of primary amines via a wide range of aldehydes (more than 52 examples), ketones (more than 26 examples), and nitriles (more than 18 examples) were proved to be broadly effective (but not all of them are shown). And also, subsequent synthesis of $\mathrm{N}, \mathrm{N}$-dimethylamine compounds following a brand-new tandem pot process by the same Co-based catalyst were simultaneously explored. In most cases, the Co-based catalyst enables easy substitution of $-\mathrm{NH}_{2}$ moiety towards functionalized and structurally diverse molecules under very mild industrially viable and scalable conditions, providing cost-effective access to numerous amines and other precursors for more complex drug products. More surprisingly, the Co-based catalyst can also be easily recycled due to its intrinsic magnetism and still remains excellent catalytic activity and selectivity for the target products even after reusing up to twelve times. Results from analytic technologies, including but not limited to XRD, XPS, TEM/Mapping and in-suit FTIR, reveals that the structural features of catalyst are closely in relation to its catalytic mechanisms; in simple terms, the outer graphitic shell is activated by the electronic interactions between the inner metallic nanoparticles and the carbon layer as well as the induced charge redistribution. Except for the gram-scale batch test in laboratory, we have firstly evaluated the lifecycle of Co-based catalyst on an automatic hydrogenation reactor by employing continuous flows of reactant ( 5 $\mathrm{ml} / \mathrm{min}$, react within $1.6 \mathrm{~min}$ ) under the similar conditions $\left(90^{\circ} \mathrm{C}, 3 \mathrm{MPa} \mathrm{H}_{2}, 0.1 \mathrm{~mol} / \mathrm{L}\right)$, whose results suggests the theoretically minimal lifecycle of Co-based catalyst can reach a continuous period of $72 \mathrm{~h}$ without 
any significant loss of catalytic efficiency. In conclusion, the advantages of this newly developed method involving operational simplicity, high stability, easily recyclable, cost-effective of the catalyst, and good functional group compatibility for the synthesis of functional amines, as well as the highly efficient and industrial applicable tandem synthesis process.

\section{Background}

Among varied kinds of chemicals, amines represent highly valuable compounds widely applied in many industry areas, including polymers, dyes, pigments, agrochemicals as well as pharmaceuticals ${ }^{1,2}$. As an example, the majority of top-selling drugs commonly contain amino groups that constitutes integral parts and plays a vital role in their application ${ }^{3}$. Hydrogenation of nitro compounds used to be the primary method for the synthesis of amines in a long period ${ }^{4}$, and several alternative methods with higher efficient have been subsequently developed, involving the alkylation of halides with $\mathrm{NH}_{3}{ }^{5,6}$, the reduction of nitriles ${ }^{7,8}$, the amination of alcohols ${ }^{9,10}$, and the reductive amination of carbonyl compounds ${ }^{11,12}$. Unfortunately, most of catalysts known for these reactions are heterogeneous but usually relies on precious metals, whose limited availability and consequently the unacceptable price as well as the toxicity of some derivatives of noble metals have spurred interest towards the development of alternative earth abundant metal catalysts ${ }^{13}$. It is thus highly important to seek an ideal procedure for the preparation of heterogeneous, non-noble, and metal-based catalysts for the advancement of effective and economic synthesis of primary amines under mild conditions.

In general, a heterogeneous catalyst composes of modified support and active phase (metal or metal oxides), where the design of functional support has recently received intense scientific interest in relevant studies for better dispersion and protection of metallic nanoparticles ${ }^{14,15}$. Compared with inorganic supports, carbon materials are superior as it offers advantages such as high stability, high surface area and abundant porous structure $^{16}$. Some of strategies have also been adopted to enhance the interaction of metallic nanoparticles with carbon materials like an encapsulated structure which coats with organic molecules, polymers or oxides, thereby preventing the leaching of catalytic metal from carbon materials ${ }^{17,18}$. For instance, Sun et al. ${ }^{19}$ reported that Co-based nanoparticles can be protected against agglomeration and air oxidation by capping with organic ligands. These coatings possess poor electron conductivity and can inevitably block charge transfer between catalytic site and reagents, so that the catalytic activity and selectivity are negatively affected. In contrast, Deng et al. ${ }^{20}$ and Chen et al. ${ }^{21}$ found that graphite-like carbon coatings with unique chemical, electrical, and functional properties are capable of protecting transition metal (e.g. Co, Fe, Ni) nanoparticles from agglomeration and re-oxidation by air, thereby preserving high activity and selectivity in a variety of catalytic reactions. In the recent, the group of Beller ${ }^{3,13}$ developed two novel heterogeneous catalysts in which Co- 
based nanoparticles are embedded in a MOF-derived precursor (acted as a structure-directing template for pyrolysis on carbon) and N-doped carbon matrix (via direct carbonization of non-volatile Co-amine coordination complexes), respectively. These Co-based catalysts exhibit outstanding activity in the reductive amination of different aldehydes and ketones along with great chemical selectivity to the corresponding substituted amines. However, although Co is claimed to be significant important in this system, the reasons behind such high activity still remain elusive, largely because of the complexity of catalyst system. Cheng et al. ${ }^{22}$ suggested that those exposed Co-based nanoparticles does not play a role in the catalytic activity, and proposed that the real active sites are highly dispersed active species located at the carbon matrix. Moreover, Liu et al. ${ }^{23}$ suggested that there is no detectable catalytic activity after acid leaching in a carbon-supported Cobased catalyst, indicating that the reductive activity is to be attributed to those accessible metallic nanoparticles. Similarly, Wang et al. ${ }^{24}$ neither detected any catalytic activity after washing Co with acid aqua on a Co-based catalyst using ZIF-67 as template. On the other hand, along the already mentioned issues in the identification of active sites in Co-based catalysts, recycling is also confronted with great challenges; indeed, most of these metallic catalysts, especially transition-metal catalyst, tended to loss their catalytic activity within a couple of runs. Consequently, an easier system of heterogeneous transition-metal catalyst preparation is necessary for the synthesis process of amines, which sever for dual purposes involving the exploration of catalytic mechanism as well as the optimization of synthesis procedure for wider application.

Herein, we describe an environmentally friendly and simplified procedure for the preparation of porously graphitic spheres that encapsulate uniform Co-based nanoparticles (Co@C) by adopting cobalt acetate as the precursor. This strategy originates from the "chainmail for catalyst" provided by the group of $\mathrm{Bao}^{20,25}$, who elaborate the unique electron penetration through the graphene layer from the encapsulated metals to promote relevant catalytic reaction on the outermost surface of graphene. In contrast to previous studies, we avoid the use of hazardous solvent and meanwhile reduce the number of synthetic steps. The most outstanding advantage of this method is undoubtedly the precise regulation of the properties of catalytic ability by modifying aqueous environment to improve metal dispersion, which is proved to be easily handle and repeatable. In this procedure, the carbonaceous precursor and graphene encapsulated metallic nanoparticles are better defined, when compared to relevant studies whose heterogeneous materials are prepared by impregnation or immobilization. Next, the obtained catalyst was applied to the challenging reductive amination of carbonyl compounds as well as reduction of nitriles for the synthesis of primary amines. We use ammonia dissolved in $\mathrm{MeOH}$ as $\mathrm{N}$ source, and the process operates under very mild conditions (90-120 ${ }^{\circ} \mathrm{C}$ and 2-4 $\mathrm{MPa} \mathrm{H}_{2}$ pressure, $4 \mathrm{~h}$ ) as common conditions for most of the reductive amination of carbonyl compounds as well as the reduction of nitriles. Using this facile synthesis of the graphene-shelled Co-based catalyst, and starting from inexpensive, readily available reactants (i.e., aldehydes, ketones, and nitriles) and molecular ammonia, we conducted the synthesis 
of more than 92 functionalized, structurally diverse linear and branched benzylic, heterocyclic, and aliphatic amines. We have also demonstrated the scale-up of the heterogeneous amination protocol to gram-scale synthesis in batch reactor and lifecycle performance via an automatic hydrogenation reactor. Last but not least, subsequent synthesis of $\mathrm{N}, \mathrm{N}$-dimethylamines products in a brand-new tandem pot process by this Co-based catalyst were simultaneously explored to enrich its catalytic functions.

\section{Preparation and characterization of Co-based catalysts}

The cobalt encapsulated in graphene-shelled catalysts were synthesized employing a simplified three-step procedure descripted in our previous work ${ }^{26}$. Briefly, we initially explored cobalt citrate (CA-Co) assembled in $\mathrm{EtOH}$ at approximate $70{ }^{\circ} \mathrm{C}$ from cobalt acetate and citric acid (CA), where the solvent was very slowly evaporated in a vacuum environment within $72 \mathrm{~h}$ in order to enable a well distribution of metallic atoms. The CA-Co could be coagulated like a sparkling jelly if most of solvent was escape from the mixture systems, and the obtained jelly, also defined as carbonaceous precursor, was subsequently carbonized at $600{ }^{\circ} \mathrm{C}$ for $2-3 \mathrm{~h}$ under $\mathrm{N}_{2}$ atmosphere. Afterwards, acid and $\mathrm{H}_{2} \mathrm{O}$ washing is necessary to remove the unloaded metals and readjust the $\mathrm{pH}$ of catalyst, respectively, following by a mild drying under vacuum and freeze environment, so as to produce the co-based nanoparticle encapsulated in graphene-shelled catalyst (Co@C-600-EtOH). It is also worthwhile mentioning that the selection of solvent absolutely affects the catalytic properties of heterogeneous catalyst because of the aqueous dispersion or polarity ${ }^{13,27}$, so that pure $\mathrm{H}_{2} \mathrm{O}$ was adopted as another solvent as a comparison. Cobalt source from varied compounds and pyrolysis temperature also functioned in different ways on catalyst properties; for example, the former might affect the chemical states of cobalt atoms, while the latter was in relation to the carbonized degree and its structural features. Furthermore, some of $\mathrm{Co} @ \mathrm{C}-600-\mathrm{EtOH}$ were oxidized under $\mathrm{O}_{2} / \mathrm{Ar}\left(5 \% \mathrm{O}_{2}\right.$ in $\mathrm{Ar}$, 99.999\% purity) atmosphere at $200{ }^{\circ} \mathrm{C}$ for $3 \mathrm{~h}$ to distinguish the catalytic properties between metallic atoms and oxides. All of these influences associated with the preparation of catalyst have been primarily discussed in this work.

Subsequently, we undertook a detailed structural analysis of these graphene-shelled nanoparticle catalysts. As except, the scanning electron microscopy (SEM) images for the Co@C-600-EtOH samples (Fig.1a) exhibited sponge-like porous feature of a carbon-supported materials, while that of catalyst carbonized at $500{ }^{\circ} \mathrm{C}$ showed a relative unbroken structure with smoother surface. The Co@C-700-EtOH sample that was prepared at a higher temperature began to collapse into smaller particles with large pores. The $\mathrm{Co} / \mathrm{CoO} @ \mathrm{C}$ 600 -EtOH sample prepared under an oxidant atmosphere at $200{ }^{\circ} \mathrm{C}$ for $3 \mathrm{~h}$ formed an interesting cubic crystal which might be the structural feature of cobalt oxide. EDS results (Fig.1a) between catalysts with or without oxidations strongly confirmed the tendency that oxygen atoms located on the surface of $\mathrm{Co} / \mathrm{CoO} @ \mathrm{C}-600$ EtOH is superior to that of Co@C-600-EtOH, implying an alternation of catalytic mechanism caused by the 
chemical forms of active $\operatorname{sites}^{3,28}$. Moreover, the high-resolution transmission electron microscopy (HRTEM) images of Co@C-600-EtOH indicated that the metallic nanoparticles were completely coated by graphene shells with an average size of $3.4 \mathrm{~nm}$. A well-resolved fringes with an interplanar spacing of $0.20 \mathrm{~nm}$ can also be clearly observed, which is attributed to the (111) plane $d$-spacing of the Co alloy (Fig.2b). In contrast, the metallic nanoparticles in Co/CoO@C-600-EtOH samples not only had the (111) plane of the Co alloy, but also exhibited the (110) plane of the $\mathrm{CoO}$ alloy with a $d$-spacing of approximate $0.22 \mathrm{~nm}$. These crystal plane exactly corresponds with the number of diaphragm shown in their corresponding diffraction pattern. By and large, according to the statistical analysis of HRTEM, it can be deduced that the graphene shells of carbonsupported materials commonly cover the metallic nanoparticles with multilayer structures, thereby forming a similar chainmail for catalysts ${ }^{25,26}$. A high-angle annular dark-field scanning transmission electron microscopy (HAADF-STEM) image with sub-angstrom resolution further confirmed the formation of the uniform metallic nanoparticles. The corresponding energy-dispersive X-ray (EDS) maps reveal the homogeneous distribution of Co atoms over the particle surface of Co@C-600-EtOH (Fig. 2c-f), whereas that of Co/CoO@C-600-EtOH suggested a higher concentration of oxygen species which are well coexisted with Co atoms.

X-ray diffraction (XRD) has been widely accepted for the determination of crystalline structure in carbonaceous materials, while Raman spectroscopy has also been established as a unique technique in probing the microstructure of carbon-based supporter since Tuinstra and Koeniget first correlated Raman bands to structure parameters calculated from XRD in $1970^{29,30}$. Both of XRD and Raman function cooperatively to provide structural information for Co@C-600-EtOH sample. The XRD spectra (Fig.1g) suggested that the peaks located at $44.2^{\circ}, 51.5^{\circ}$, and $75.8^{\circ}$ represent crystal plane of (110), (200), and (220) of cobalt atoms $\mathrm{s}^{3,16}$, and the Raman spectra (Fig.1h) confirmed the higher degree of aromatization and crystallinity by showing two essential peaks around $1590 \mathrm{~cm}^{-1}$ ( $\mathrm{G}$ band, indicator of graphite) and $1350 \mathrm{~cm}^{-1}$ (D band, indicator of defect), respectively ${ }^{30}$. Another analysis of X-ray photoelectron spectroscopy (XPS) indicated the presence of both metallic Co $\left(2 \mathrm{p}_{3 / 2}\right)$ and $\mathrm{Co}\left(\mathrm{Co} 2 \mathrm{p}_{1 / 2}\right)$ species on the catalyst surface (shown in Fig. $\left.1(\mathrm{j})\right)^{16}$. Moreover, the nitrogen physical adsorption for synthesized carbonaceous materials in the specific surface area (BrunauerEmmett-Teller method, shown in Fig.1(i)) were proved to be in accordance with the porous structures exhibited in SEM images. At a higher pyrolysis temperature, Co@C-700-EtOH has a larger surface area (997 $\left.\mathrm{cm}^{2} / \mathrm{g}\right)$ and pore volume $(1.79 \mathrm{cc} / \mathrm{g})$ that that of Co@C-600-EtOH $\left(475 \mathrm{~cm}^{2} / \mathrm{g}, 0.91 \mathrm{cc} / \mathrm{g}\right)$ and Co@C-500$\mathrm{EtOH}\left(408 \mathrm{~cm}^{2} / \mathrm{g}, 0.80 \mathrm{cc} / \mathrm{g}\right)$. If the oxidation step was taken into consideration, the relevant parameters were slightly decreased to $359.2 \mathrm{~cm}^{2} / \mathrm{g}$ and $0.69 \mathrm{cc} / \mathrm{g}$ (in Co/CoO@C-600-EtOH) caused by the overlap of metal oxides in structural pores. Following with the distribution of BET curves, the identical pore sizes were mainly located at a range within $2-5 \mathrm{~nm}$, coinciding with the average sized of metallic nanoparticles observed in TEM images. These results indicated that uniform Co nanoparticle have been well encapsulated in thin and 
multilayer graphene shells.
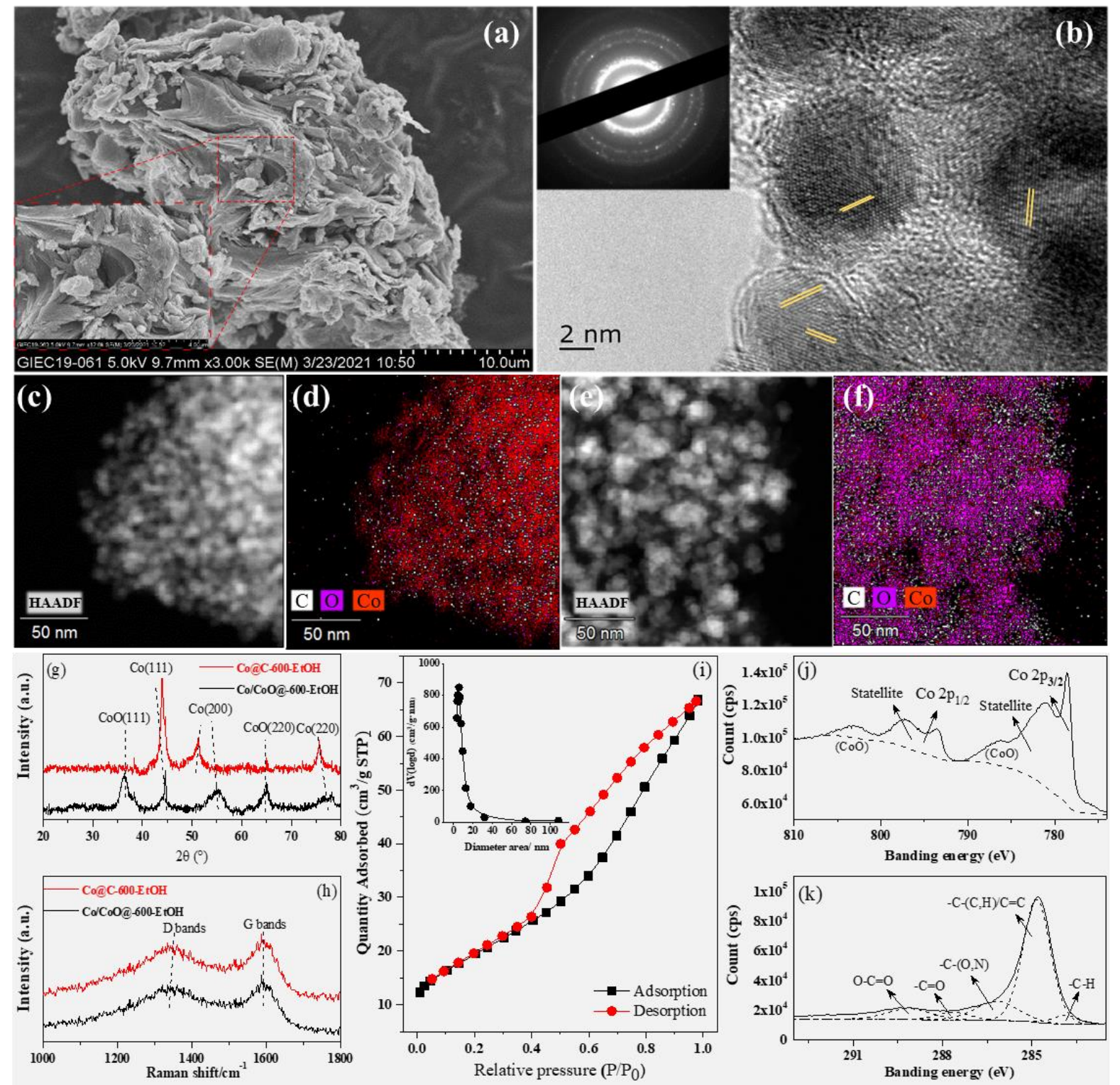

Fig.1 Catalyst characterization. SEM image of Co@C-600-EtOH (a), TEM image of Co@C-600-EtOH (b), and insets in here show the selected-area of surface features in SEM and electron diffraction pattern in TEM; HAADF-TEM images of a single particle in $\mathrm{Co} @ \mathrm{C}-600-\mathrm{EtOH}$ (c) and Co/CoO@C-600-EtOH (e) with the corresponding EDS element mapping of C, O, and $\mathrm{Co}$ in $\mathrm{Co} @ \mathrm{C}-600-\mathrm{EtOH}(\mathrm{d})$ and Co/CoO@C-600-EtOH (f); the results of analytic technologies including XRD (g), Raman (h), BET (i), and XPS spectra of Co and C (j-k) were conducted for Co@C-600-EtOH.

\section{Synthesis of primary amines}

All of these prepared catalysts as well as some of commercial catalysts were tested in the benchmark reductive amination of benzaldehyde by employing $7 \mathrm{M} \mathrm{NH}_{3}$ in $\mathrm{MeOH}$ solution and $2 \mathrm{MPa} \mathrm{H}_{2}$ to produce benzylamine, which is presented as a structural motif in several bioactive molecules ${ }^{2,27,28}$. Based on the primary experiments as shown in Table 1, the change of pyrolysis temperatures in the preparation of catalysts strongly affects its catalytic selectivity for $1 \mathrm{a}$ in the reduction amination of benzaldehyde (Entry 1-3) suggesting that an ideal temperature of $600{ }^{\circ} \mathrm{C}$ is recommended. The use of EtOH can also improve the catalytic selectivity for $1 \mathrm{a}$ when compared to the catalysts prepared in $\mathrm{H}_{2} \mathrm{O}$ (Entry 2, 4). Several sources of 
cobalt compounds involving cobalt acetate, cobalt carbonate, and cobalt nitrate indicated similar effects on catalytic activity and selectivity. However, the introduction of $\mathrm{CoO}$ during oxidation step significantly decreased the catalytic selectivity for 1a (Entry 5). It can be concluded that the best catalyst according to our experimental procedure is $\mathrm{Co@C-600-EtOH,} \mathrm{whose} \mathrm{catalytic} \mathrm{activity} \mathrm{and} \mathrm{selectivity} \mathrm{to} \mathrm{yield} \mathrm{1a} \mathrm{reach} \mathrm{more}$ than $99 \%$ and $96 \%$, respectively. By contrast, most of noble-based catalysts (i.e., $5 \% \mathrm{Ru} / \mathrm{C}, 5 \% \mathrm{Pt} / \mathrm{C}, 5 \%$ $\mathrm{Pt} / \mathrm{C}$, and $5 \% \mathrm{Pd} / \mathrm{C}$ ) exhibited a lower selectivity for reductive amination (Entry 7-10), and the use of commercial Raney Co only afforded a 78.9 \% yield of 1a while Raney Ni yield 32.8 \% (Entry 11,12). Form these results, the reductive amination can be successfully done by using the Co@C-600-EtOH sample under mild reaction conditions. Other significant influences on the yield of $1 \mathrm{a}$ are relevant to the reaction conditions, including the reaction temperature, holding time, solvent concentration, and $\mathrm{H}_{2}$ pressure ${ }^{3,31,32}$. In summary, the reductive amination catalyzed by $\mathrm{Co} @ \mathrm{C}-600-\mathrm{EtOH}$ can reach its best result under the optimized conditions: $90{ }^{\circ} \mathrm{C}, 4 \mathrm{~h}, 10 \mathrm{mg}$ catalyst, $5 \mathrm{ml}$ ammonia solution $\left(7 \mathrm{M} \mathrm{NH}_{3}\right.$ in $\left.\mathrm{MeOH}\right)$, and $2 \mathrm{MPa}_{2}$.

Table 1 Optimization of catalyst preparation and reaction conditions for reductive amination

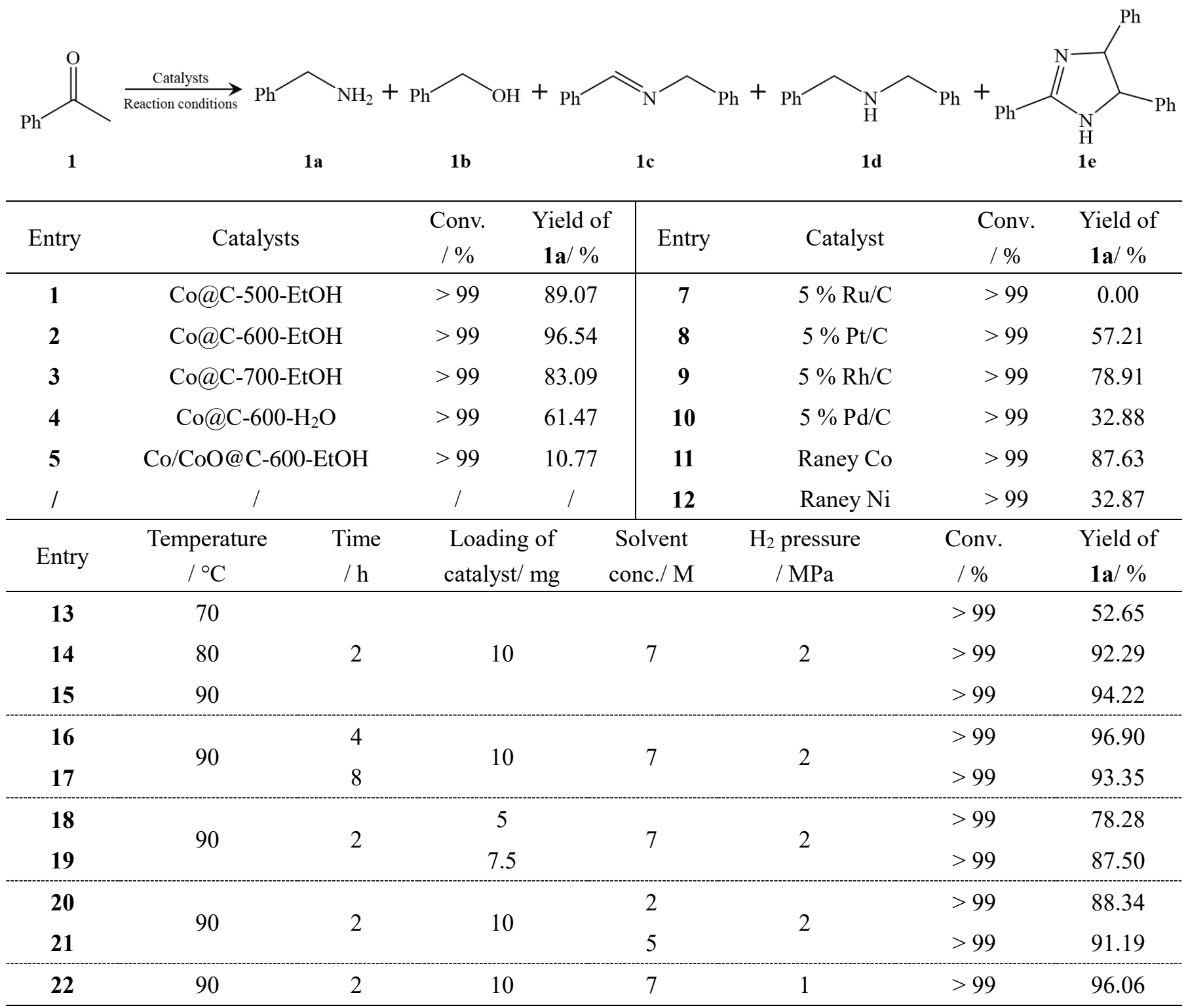


Note: Screening of prepared and commercial catalysts are based on the below conditions: $80^{\circ} \mathrm{C}, 2 \mathrm{~h}, 10 \mathrm{mg}$ catalyst, $5 \mathrm{ml}$ ammonia solution (7 M NH 3 in $\mathrm{MeOH}$ ), $2 \mathrm{MPa} \mathrm{H}_{2}$, and $0.5 \mathrm{mmol}$ benzaldehyde; the better catalyst of Co@C-600-EtOH was selected for the optimization of reaction conditions. Yields and conversion were determined by GC-MS and ${ }^{1} \mathrm{H}$ NMR spectroscopy using 1,3,5-trimethoxybenzenen as an internal standard.

After selecting the suitable catalyst and the optimized reaction conditions for benchmark reaction, we then explored the substrate scope of catalyst in catalyzing a wide range of aldehydes, ketones and nitriles for the synthesis of primary amines via reductive or reduction amination, which can easily be functionalized further and thus serve as central building blocks. First of all, we applied the Co@C-600-EtOH sample under mild reaction conditions $\left(90{ }^{\circ} \mathrm{C}, 2 \mathrm{MPa} \mathrm{H}_{2}, 4 \mathrm{~h}\right)$ in the selective reductive amination of 38 aldehydes to produce benzylic, heterocyclic, and aliphatic linear primary amines, in good to excellent yields (up to $99 \%$ ). As shown in Fig.2, most of the aromatic aldehydes (Entry 24) or its derived compounds substituted by methyl (Entry 2528), halogenated (Entry 28-34), and methoxyl (Entry 35-37) groups were efficiently converted into the corresponding products with good to excellent yield. On another hand, the reductive amination of aliphatic aldehydes had been reported to be impeded by unproductive aldol condensation which occurs under basic conditions $^{26}$; even so, we obtained the excellent yields (up to $99 \%$ ) and high selectivity for the synthesis of primary amines from most of aliphatic aldehydes (Entry 43-51). More interestingly, the typical biomassderived compounds can also be converted to the corresponding products with excellent yield (Entry 36$38)^{13,27,28}$, except for vanillin. Other functional groups such as furan, methyl-furan, 5-hydroxymethyl furfural, pyridine, and amide were also tolerated in our experiments (Entry 52-61).

Similarly, the same reaction condition was applied in the reductive amination of ketones, which is a much faster route than the reduction of the corresponding ketone-derived imines ${ }^{33,34}$. We were pleased to find that an efficient synthesis of primary amines from both of the industrially relevant and structurally challenging ketones was achieved, together with satisfied yields of the corresponding primary amines. Biologically active amphetamines $^{13}$, which are potent central nervous system (CNS)-stimulating drugs, were synthesized in a well yield up to $95 \%$. Other aromatic and heterocyclic ketones were tolerated well (Entry 64, 66, 68-70,73,74), while purely aliphatic ketones also endured the reaction conditions to produce the corresponding primary amines in excellent yields with even the cycloalkyl ketones having varied ring sizes being successfully selectively aminated (Entry 71,72). Substrates containing nitrile groups were further introduced, and the corresponding primary amines were obtained in good yields (Entry 75-85) although the reaction conditions are harsher $\left(120{ }^{\circ} \mathrm{C}, 4 \mathrm{MPa}, 8 \mathrm{~h}\right)$. The conversion of electron-rich methyl-/methoxy-substituted aryl-alkyl nitriles occurs sufficiently, and these fluoride (Entry 80, 81, 84), bromine (Entry 83), and chloride (Entry 82, 85) substrates are also tolerated well ${ }^{7,8}$. To explore the potential activity of the existing bioactive molecules, these compounds need to be functionalized or the functional groups must be varied. So far, the introduction 
of amino groups into these bioactive molecules has not been extensively discussed. According to our experimental results, most of the carbonyl and nitrile compounds were easily and selectively converted to their corresponding primary amines by employing this catalyst system. These syntheses showcase the applicability of the carbon-supported Co-based nanoparticles for the selective preparation of precursor for the aminederived drugs.

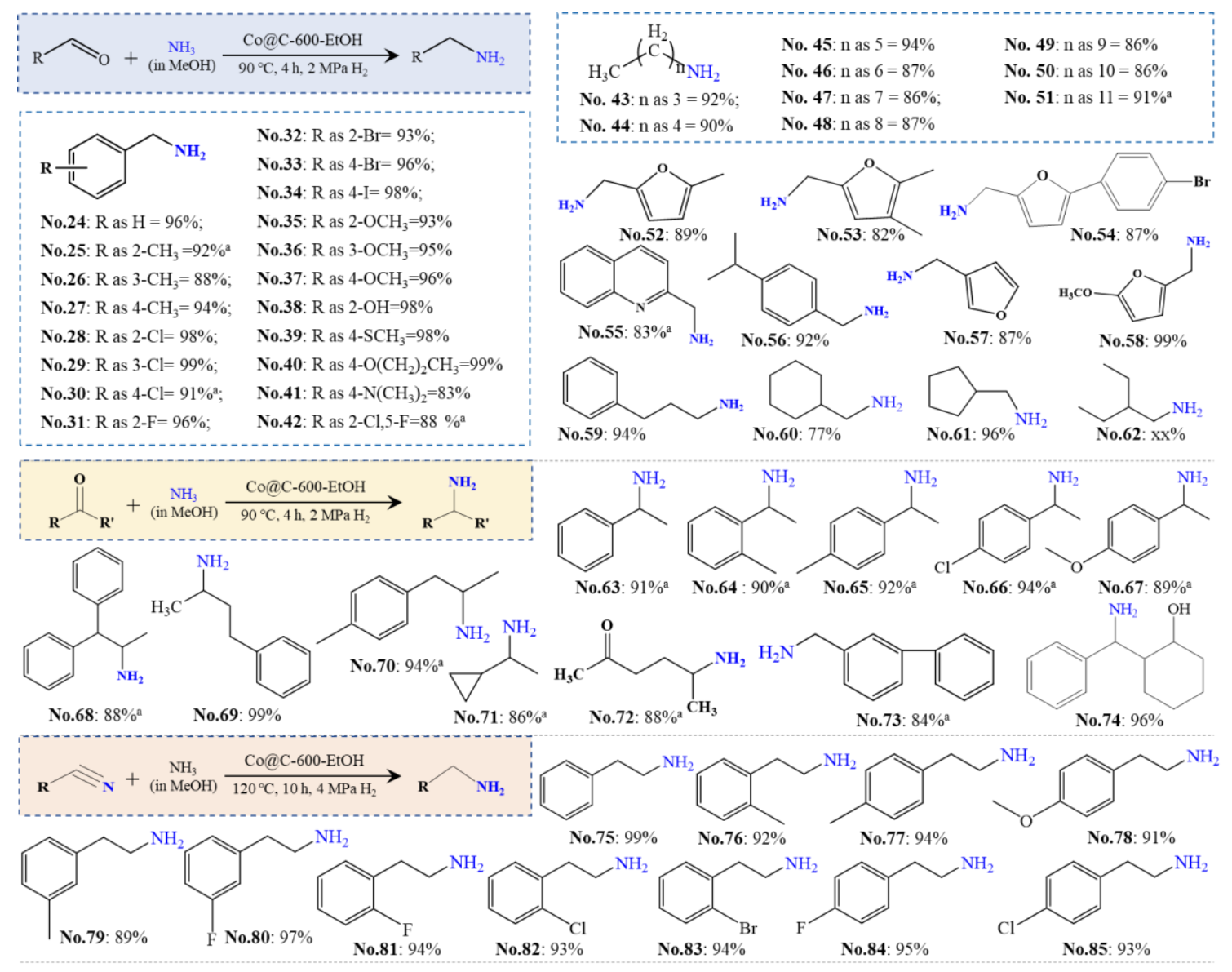

Fig.2 Co@C-600-EtOH-catalyzed reductive amination of aldehydes, ketones, and nitriles for the synthesis of primary amines. Reaction conditions: 0.5 mmol reactants, 10 mg Co@C-600-EtOH catalyst, $5 \mathrm{ml}$ ammonia solution (7 M NH3 in methanol), and the preset temperature, time and $\mathrm{H}_{2}$ pressure shown in formulas. ${ }^{a}$ Reaction conditions were elevated to

$100{ }^{\circ} \mathrm{C}, 2 \mathrm{MPa} \mathrm{H}_{2}, 4 \mathrm{~h}$. Yields and conversion were determined by GC-MS and ${ }^{1} \mathrm{H}$ NMR spectroscopy using 1,3,5trimethoxybenzenen as an internal standard.

\section{Synthesis of N, N-dimethylamines}

Encouraged by the successful synthesis of primary amine with the help of Co@C-600-EtOH catalyst, we kept on developing another more cost-effective and highly practical tandem synthesis of tertiary amines which originates from the easily accessible aromatic aldehydes and $\mathrm{NH}_{3}{ }^{2,21}$. This method shows significant benefits both on efficiency and economics as the synthesis of secondary and tertiary amines that are found in many biologically active natural products ${ }^{26}$. In detail, we combined the reductive amination of aldehydes and Nmethylation of the obtained primary amines into a one-pot tandem synthesis process as depicted in Fig.3, by using the only catalyst of $\mathrm{Co} @ \mathrm{C}-600-\mathrm{EtOH}$ without any purification. Excellent yields of N-diethylation products, usually detected as the tertiary amines (i.e., N, N-dimethylamines), were observed for all the test 
para-substituted aromatic aldehydes (Entry 86-88), ketones (Entry 89-91), and nitriles (Entry 92-94). This process is cost-efficient, easily separation, high harvest of the desired products and inhibits the formation of by-product which matches the sustainable and green chemistry very well.
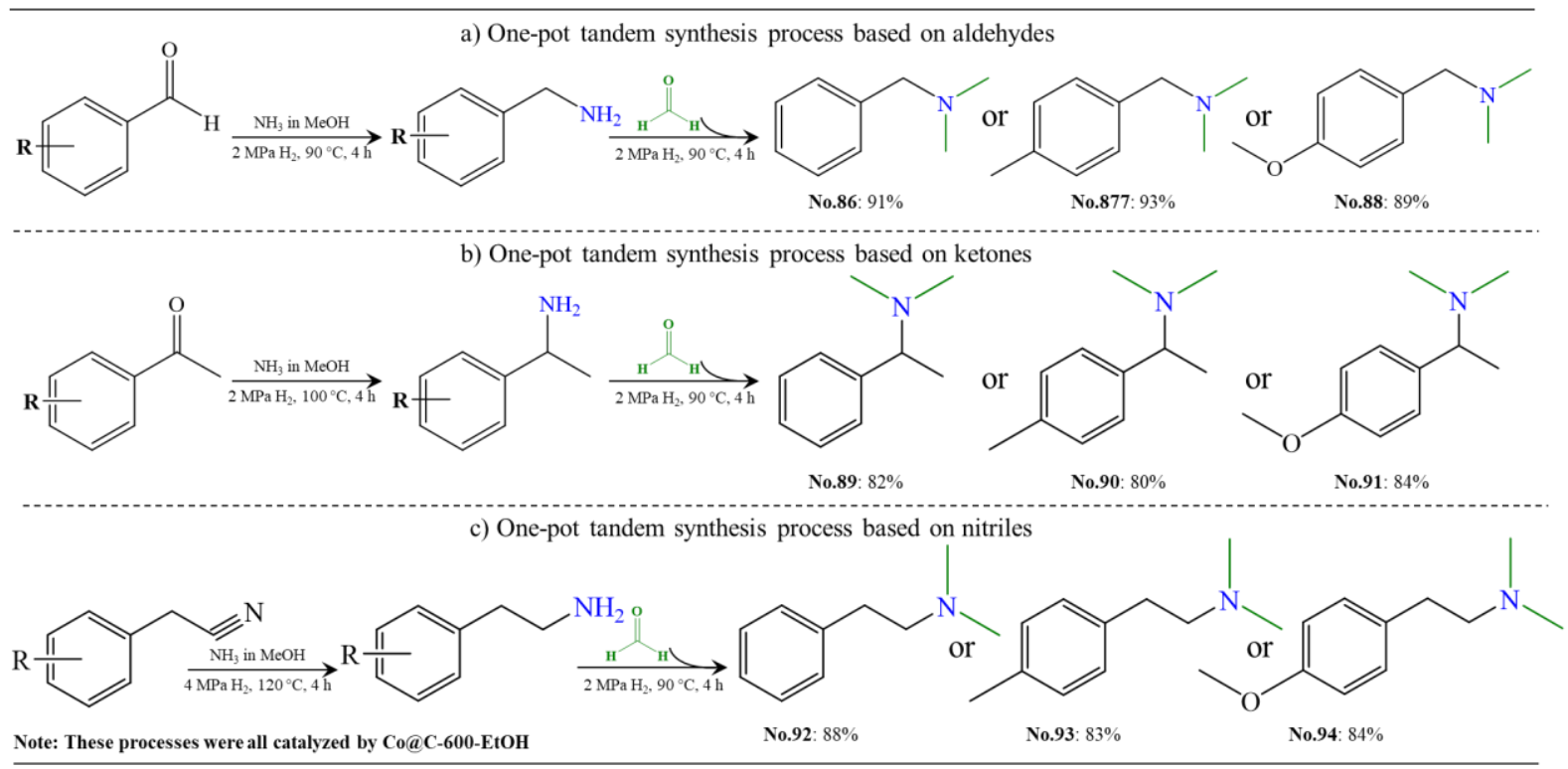

Fig.3 Co@C-600-EtOH-catalyzed synthesis of N, N-dimehylamines. Reaction conditions: 10 mg Co@C-600-EtOH catalyst, $0.5 \mathrm{mmol}$ reactant, $5 \mathrm{ml}$ ammonia solution ( $7 \mathrm{M} \mathrm{NH}_{3}$ in methanol), and the preset temperature, time and $\mathrm{H} 2$ pressure shown in formulas; after the reaction was completed, $3 \mathrm{mmol}$ formaldehyde ( $37 \%$ aq) was added for the subsequent reaction. Yields and conversion were determined by GC-MS and ${ }^{1} \mathrm{H}$ NMR spectroscopy using 1,3,5trimethoxybenzenen as an internal standard.

\section{Gram-scale reactions and recycling of the catalyst.}

After successful synthesis of primary amines via the reductive amination and tertiary amines via the onepot tandem synthesis process, we next plan to apply this system in the future industry. And here we were delighted to show three substrates were well performed in this reductive amination at the gram scale, as shown in Fig.4. Reductive aminations having a 20 times higher amount of the substrates $(10 \mathrm{mmol})$ were performed to demonstrate the applicability of the novel catalyst system for gram-scale syntheses. Under the optimal conditions, they were similar to those small-scale reactions, and excellent yields of the target product were obtained for all substrates (Entry 24, 27, 37). Furthermore, the most essential characteristics for any industrial catalysts are the stability and recyclability ${ }^{17,18,20,25,32}$. Apart from the apparent cost advantages associated with these characteristics and the ease of accessibility, the use of easily recyclable heterogeneous catalysts would remarkably support in product purification. For this reason, we then evaluated the catalyst stability and recyclability using the benchmark reaction under the suitable catalyst (i.e., CO@C-600-EtOH) and optimized reaction conditions $\left(90^{\circ} \mathrm{C}, 2 \mathrm{MPa} \mathrm{H}_{2}, 4 \mathrm{~h}\right)$. Surprisingly, in contrast to other commercial or previously reported catalysts, our graphene-shelled metallic catalyst exhibits great stability. This catalyst could be immobilized on a magnetic stirring bar and be conveniently recycled up to twelve times still having high product selectivity 
and catalyst activity (Fig. 4), as concluded from the initial rate measurements.

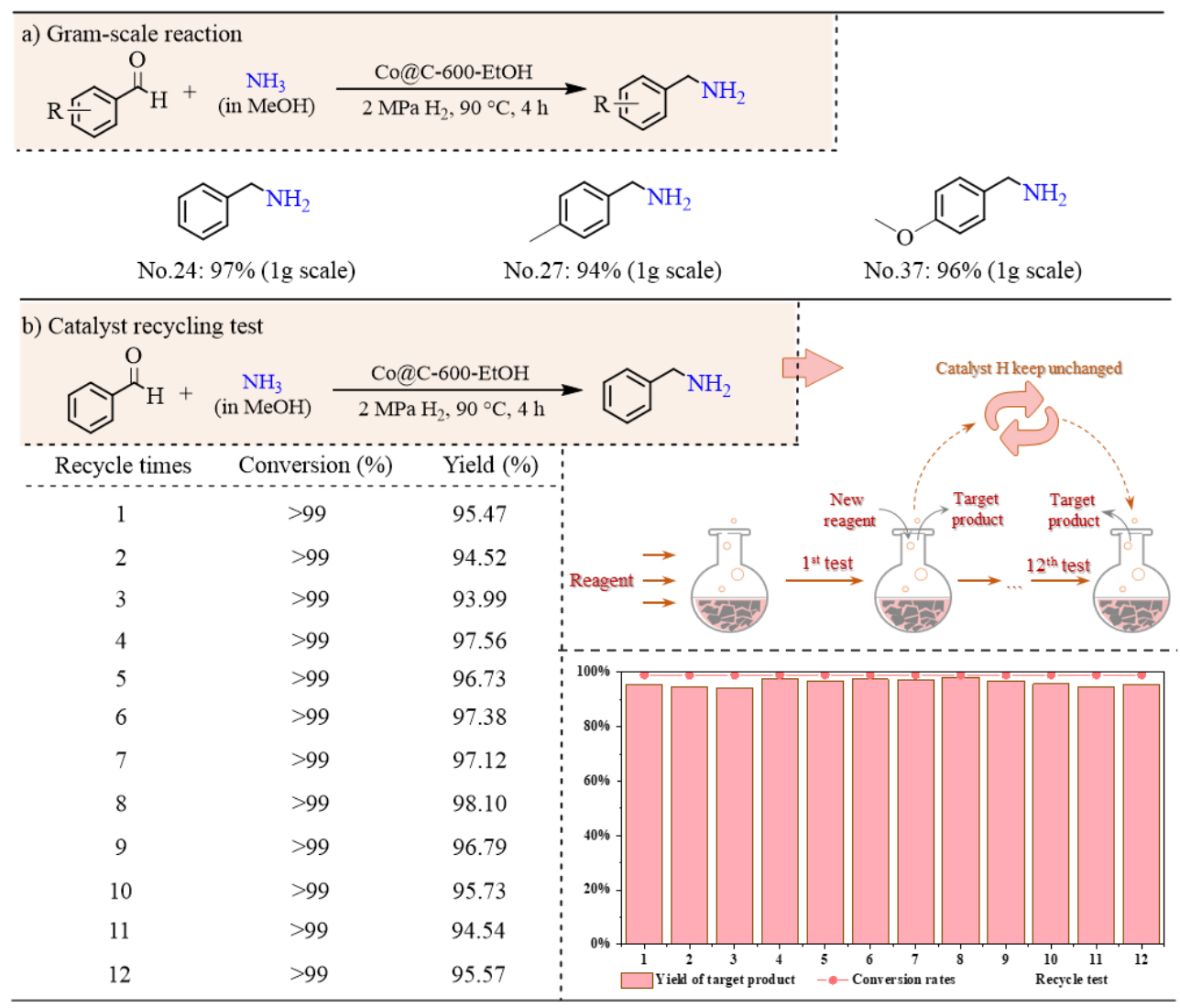

Fig.4 Gram-scale reaction and recycling of the catalyst. a) Gram-scale reaction conditions: 200 mg Co@C-600-EtOH catalyst, $40 \mathrm{mmol}$ benzaldehydes, $100 \mathrm{~mL}$ ammonia solution ( $7 \mathrm{M} \mathrm{NH}_{3}$ in methanol), $2 \mathrm{MPa} \mathrm{H}_{2}, 90{ }^{\circ} \mathrm{C}, 4 \mathrm{~h}$; b) Catalyst recycling test reaction conditions: $10 \mathrm{mg}$ Co@C-600-EtOH catalyst, $0.5 \mathrm{mmol}$ benzaldehyde, $5 \mathrm{~mL}$ ammonia solution $(7 \mathrm{M}$

$\mathrm{NH}_{3}$ in methanol), $2 \mathrm{MPa} \mathrm{H}, 90{ }^{\circ} \mathrm{C}, 4 \mathrm{~h}$. Yields and conversion were determined by GC-MS and ${ }^{1} \mathrm{H}$ NMR spectroscopy using 1,3,5-trimethoxybenzenen as an internal standard.

\section{Automatic reactor for uninterrupted reaction of reductive amination}

So far, the benchmark tests in laboratory scale have been widely studied but also remains some obstructions when taking the industrial application into account. With the help of recently developed techniques, we can now employ an automatic hydrogenation reactor to conduct the uninterrupted process of reductive amination for the synthesis of primary amines in $\mathrm{g}$-scale/h, which can evaluate the applied potentials of catalysts to a certain extent. Technically, this automatic reactor mainly consists of three important sections: a high-pressure infusion pump, a reaction chamber, and a gas-liquid separator. At first, the reaction chamber is filled with approximate1.2 g Co@C-600-EtOH samples that were pretreated to form 0.5-1 cm pellet, and the chamber environment is maintained at $90{ }^{\circ} \mathrm{C}$ and $3 \mathrm{MPa}$ reductive atmosphere with $50 \mathrm{ml} / \mathrm{min}$ flow of $\mathrm{H}_{2}$. Subsequently, the reactant flow with a concentration of $0.1 \mathrm{~mol} / \mathrm{L}$ is pumped into the reaction chamber in forms of gas-liquid mixture; the flow rate was controlled between $0.5-5 \mathrm{ml} / \mathrm{min}$, which can be used to calculated the actual reaction period as the chamber volume is around $8 \mathrm{~cm}^{3}$. More importantly, the change of reactant forms improves the interaction between reactant and catalyst, which support the rapid and sufficient process of reductive 
amination within 1.6-16 min. And then, the mixture flow is passed by a gas-liquid separator to purify and collect the target products in a constant rate. As can be seen in Fig.5, the synthesis of primary amines via reductive amination is comparable to the benchmark test, showing an excellent yield of target products even at a flow of $5 \mathrm{ml} / \mathrm{min}$ (up to $97 \%$ ), which indicates that only $1.6 \mathrm{~min}$ is adequate for the completely conversion of benzaldehyde in this automatic reactor. A long-term performance of reductive amination up to 150 min (at a constant reactant flow of $3 \mathrm{ml} / \mathrm{min}$ ) will not reduce the catalytic activity and selectivity, which can be theoretically calculated to produce benzylamine with a scale of 46 g/day based on the lifecycle of Co@C-600EtOH shown in Fig.4. In fact, the amount of catalyst used in here is overload, thus much higher concentration of reactant flow or prolonging the runtime are next goals to obtain the maximum efficiency of reductive amination of the synthesis of primary amines. This innovative technique suggests an incredible advantage when compared to the usual benchmark test in most of reported studies as it can reduce the reaction time and optimize the synthesis mode, providing an efficient and economic strategy for the synthesis of high-value platform for medical drugs or other chemicals. Encouraged by this experience, we have currently applied this highly active, stable, easily hand-able and low-cost Co-based catalyst on another larger automatic reactor which enables the synthesis of primary amines in kg-scale/day or ton-scale/year.

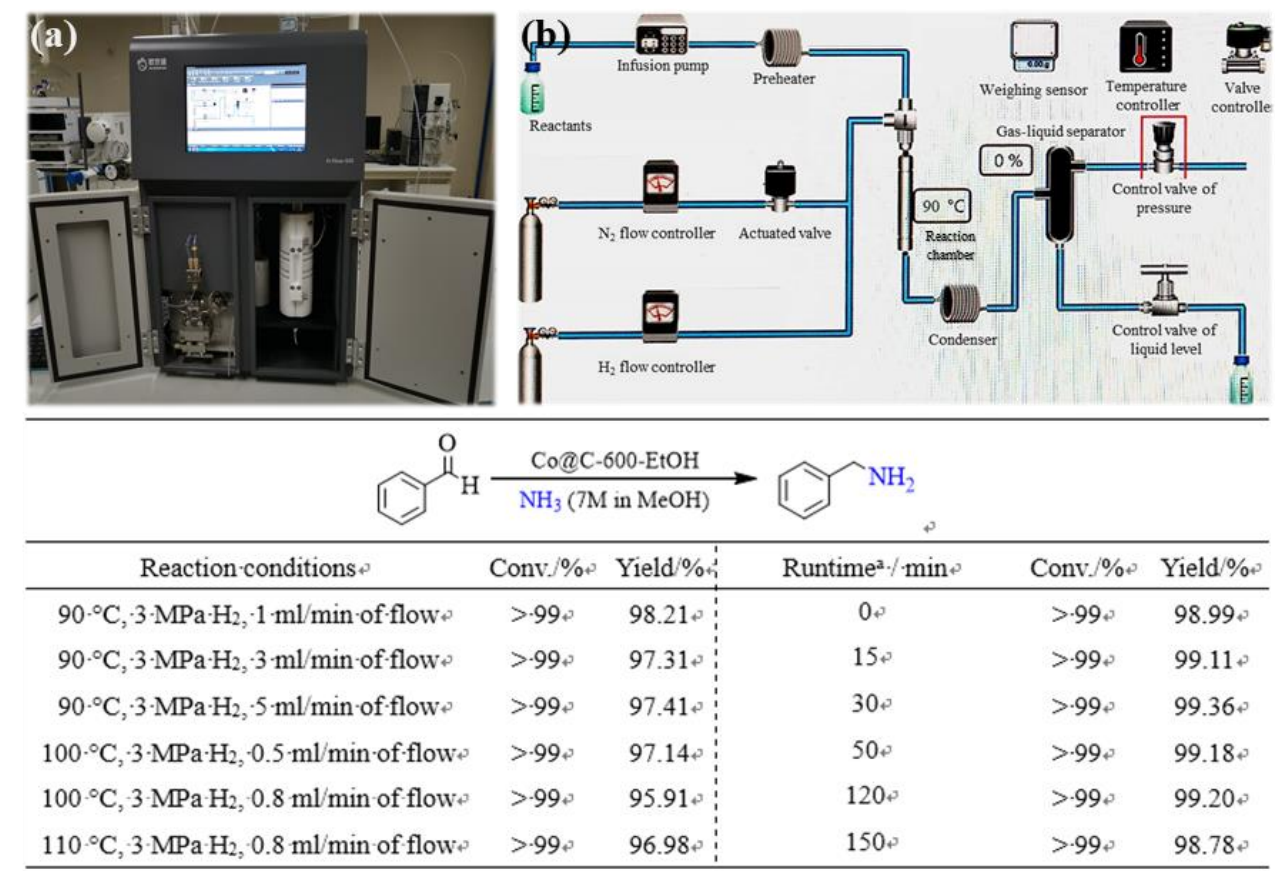

Fig.5 The appearance (a) and the schematic diagram (b) of automatic hydrogenation reactor, together with the result of uninterrupted reductive amination based on benzaldehyde. Reaction conditions:0.1 mol/L reactants, 1.2g Co@C-600-EtOH catalyst, $200 \mathrm{ml}$ ammonia solution ( $7 \mathrm{M} \mathrm{NH}_{3}$ in methanol), and the preset temperature, time and $\mathrm{H}_{2}$ pressure shown in table. Runtime indicates the performance period of automatic reactor under $90{ }^{\circ} \mathrm{C}, 3 \mathrm{MPa} \mathrm{H}_{2}, 3 \mathrm{ml} / \mathrm{min}$ flow of reactant. Yields and conversion were determined by GC-MS and ${ }^{1} \mathrm{H}$ NMR spectroscopy using 1,3,5-trimethoxybenzenen as an internal standard.

\section{Conclusions}


In conclusion, uniform nanoparticle catalysts encapsulated in multi-layer graphene structure using cobalt acetate as the precursor are synthesized successfully following with a simple and environmentally friendly method. The catalysts coupled easily accessible a wide range of aldehydes, ketones, and nitriles with ammonia and $\mathrm{H}_{2}$ under mild industrially viable and scalable conditions $\left(90^{\circ} \mathrm{C}, 2 \mathrm{MPa} \mathrm{H}_{2}, 4 \mathrm{~h}\right)$. Various functionalized, structurally diverse linear and branched benzylic, heterocyclic, and aliphatic amines including drugs derivatives (more than 92 examples) were synthesized in good to excellent yield. More importantly, this protocol not only exhibited a similar reactivity during the gram-scale or uninterrupted synthesis of primary amines when compared to the small-scale in laboratory test, but also possessed a longer lifecycle as its can be easily recycled and reused up to twelve times without any significant loss of catalytic activity and selectivity. The successfully synthesis of N, N-dimethylamines products via reductive amination of aldehydes and Nmethylation of amines in a one-pot tandem process have also been measured, enriching the applied functions of this Co@C-600-EtOH. The above findings strongly confirm an available and the feasible preparation of simple but highly efficient catalysts towards reductive amination. By and large, the advantages of this newly developed method involving operational simplicity, high stability, easily recyclable, cost-effective of the catalyst, and good functional group compatibility for the synthesis of functional amines, as well as the highly efficient and industrial applicable tandem synthesis process.

\section{Reference}

1 Chen, X., Zhao, H., Chen, C., Jiang, H. \& Zhang, M. Transfer hydrogenative para-selective aminoalkylation of aniline derivatives with N-heteroarenes via ruthenium/acid dual catalysis. Chemical Communications 54, 9087-9090, doi:10.1039/C8CC04233K (2018).

2 Chakrabarti, K., Mishra, A., Panja, D., Paul, B. \& Kundu, S. Selective synthesis of mono- and di-methylated amines using methanol and sodium azide as C1 and N1 sources. Green Chemistry 20, 3339-3345, doi:10.1039/C8GC00863A (2018). Murugesan, K., Chandrashekhar, V. G., Senthamarai, T., Jagadeesh, R. V. \& Beller, M. Reductive amination using cobaltbased nanoparticles for synthesis of amines. Nature protocols 15, 1313-1337, doi:10.1038/s41596-019-0258-z (2020). Haydar, G., Hakan, S., Benan, K. \& Fatih, S. Recent Advances in the Reduction of Nitro Compounds by Heterogenous Catalysts. Current Organic Chemistry 21, 794-820, doi:http://dx.doi.org/10.2174/1385272820666160525123907 (2017). Oderinde, M. S. et al. Highly Chemoselective Iridium Photoredox and Nickel Catalysis for the Cross-Coupling of Primary Aryl Amines with Aryl Halides. Angewandte Chemie International Edition 55, 13219-13223, doi:https://doi.org/10.1002/anie.201604429 (2016).

6 Jung, H. S., Yun, T., Cho, Y. \& Jeon, H. B. Simple and convenient copper-catalyzed amination of aryl halides to primary arylamines using NH4OH. Tetrahedron 72, 5988-5993, doi:https://doi.org/10.1016/j.tet.2016.07.021 (2016).

Adam, R. et al. Selective Hydrogenation of Nitriles to Primary Amines by using a Cobalt Phosphine Catalyst. Chemsuschem 10, 842-846, doi:https://doi.org/10.1002/cssc.201601843 (2017). Pincer Complexes. J Am Chem Soc 138, 8809-8814, doi:10.1021/jacs.6b03709 (2016). Demidova, Y. S. et al. One-pot monoterpene alcohol amination over $\mathrm{Au} / \mathrm{ZrO} 2$ catalyst: Effect of the substrate structure. 
Journal of Catalysis 360, 127-134, doi:https://doi.org/10.1016/j.jcat.2018.01.020 (2018).

Huy, P. H., Motsch, S. \& Kappler, S. M. Formamides as Lewis Base Catalysts in SN Reactions-Efficient Transformation of Alcohols into Chlorides, Amines, and Ethers. Angewandte Chemie International Edition 55, 10145-10149, doi:https://doi.org/10.1002/anie.201604921 (2016).

Nayal, O. S., Bhatt, V., Sharma, S. \& Kumar, N. Chemoselective Reductive Amination of Carbonyl Compounds for the Synthesis of Tertiary Amines Using SnCl2·2H2O/PMHS/MeOH. The Journal of Organic Chemistry 80, 5912-5918, doi:10.1021/acs.joc.5b00156 (2015).

Mutti, F. G., Knaus, T., Scrutton, N. S., Breuer, M. \& Turner, N. J. Conversion of alcohols to enantiopure amines through dual-enzyme hydrogen-borrowing cascades. Science 349, 1525, doi:10.1126/science.aac9283 (2015).

Jagadeesh, R. V. et al. MOF-derived cobalt nanoparticles catalyze a general synthesis of amines. Science 358, 326, doi:10.1126/science.aan6245 (2017).

Seo, B. et al. Preferential horizontal growth of tungsten sulfide on carbon and insight into active sulfur sites for the hydrogen evolution reaction. Nanoscale 10, 3838-3848, doi:10.1039/C7NR08161H (2018).

Moradabadi, A., Ahmadi, S. \& Kaghazchi, P. Evidence of a strong effect of defect-free metal oxide supports on Pt nanoparticles. Nanoscale 9, 4478-4485, doi:10.1039/C6NR07816H (2017).

Yuan, Z., Liu, B., Zhou, P., Zhang, Z. \& Chi, Q. Preparation of nitrogen-doped carbon supported cobalt catalysts and its application in the reductive amination. Journal of Catalysis 370, 347-356, doi:https://doi.org/10.1016/j.jcat.2019.01.004 (2019).

Liao, Y.-T. et al. De Novo Synthesis of Gold-Nanoparticle-Embedded, Nitrogen-Doped Nanoporous Carbon Nanoparticles (Au@NC) with Enhanced Reduction Ability. ChemCatChem 8, 475-475, doi:https://doi.org/10.1002/cctc.201600023 (2016).

Chen, L. et al. A covalent organic framework-based route to the in situ encapsulation of metal nanoparticles in N-rich hollow carbon spheres. Chemical Science 7, 6015-6020, doi:10.1039/C6SC01659F (2016).

Sun, X. et al. Metal-Organic Framework Mediated Cobalt/Nitrogen-Doped Carbon Hybrids as Efficient and Chemoselective Catalysts for the Hydrogenation of Nitroarenes. ChemCatChem 9, 1854-1862, doi:https://doi.org/10.1002/cctc.201700095 (2017).

Deng, D. et al. Iron Encapsulated within Pod-like Carbon Nanotubes for Oxygen Reduction Reaction. Angewandte Chemie International Edition 52, 371-375, doi:https://doi.org/10.1002/anie.201204958 (2013).

Chen, M. et al. Highly Stretchable Conductors Integrated with a Conductive Carbon Nanotube/Graphene Network and 3D Porous Poly(dimethylsiloxane). Advanced Functional Materials 24, 7548-7556, doi:https://doi.org/10.1002/adfm.201401886 (2014).

Cheng, T. et al. Identifying active sites of CoNC/CNT from pyrolysis of molecularly defined complexes for oxidative esterification and hydrogenation reactions. Catalysis Science \& Technology 6, 1007-1015, doi:10.1039/C5CY01349F (2016).

Liu, L., Concepción, P. \& Corma, A. Non-noble metal catalysts for hydrogenation: A facile method for preparing Co nanoparticles covered with thin layered carbon. Journal of Catalysis 340, 1-9, doi:https://doi.org/10.1016/j.jcat.2016.04.006 (2016).

Wang, X. \& Li, Y. Chemoselective hydrogenation of functionalized nitroarenes using MOF-derived co-based catalysts. Journal of Molecular Catalysis A: Chemical 420, 56-65, doi:https://doi.org/10.1016/j.molcata.2016.04.008 (2016). Yu, L., Deng, D. H. \& Bao, X. H. Chain Mail for Catalysts. Angew Chem Int Edit 59, 15294-15297, doi:10.1002/anie.202007604 (2020).

Liu, J. et al. Facile synthesis of controllable graphene-co-shelled reusable Ni/NiO nanoparticles and their application in the synthesis of amines under mild conditions. Green Chemistry 22, 7387-7397, doi:10.1039/D0GC02421J (2020). 

amines. Nat Commun 9, 4123, doi:10.1038/s41467-018-06416-6 (2018). Murugesan, K. et al. Homogeneous cobalt-catalyzed reductive amination for synthesis of functionalized primary amines. Nat Commun 10, 5443, doi:10.1038/s41467-019-13351-7 (2019). Li, X. J., Hayashi, J. \& Li, C. Z. FT-Raman spectroscopic study of the evolution of char structure during the pyrolysis of a Victorian brown coal. Fuel 85, 1700-1707 (2006).

Zhuang, X. Z. et al. Insights into the evolution of chemical structures in lignocellulose and non-lignocellulose biowastes during hydrothermal carbonization (HTC). Fuel 236, 960-974, doi:https://doi.org/10.1016/j.fuel.2018.09.019 (2019). Alinezhad, H., Yavari, H. \& Salehian, F. Recent Advances in Reductive Amination Catalysis and Its Applications. Current Organic Chemistry 19, 1021-1049 (2015).

Manzoli, M. et al. Microwave-Assisted Reductive Amination with Aqueous Ammonia: Sustainable Pathway Using Recyclable Magnetic Nickel-Based Nanocatalyst. Acs Sustain Chem Eng 7, 5963-5974, doi:10.1021/acssuschemeng.8b06054 (2019).

Friedfeld, M. R. et al. Cobalt Precursors for High-Throughput Discovery of Base Metal Asymmetric Alkene Hydrogenation Catalysts. Science 342, 1076-1080, doi:10.1126/science.1243550 (2013). Hydrogenation of Ketones and Imines. Science 342, 1080-1083, doi:10.1126/science.1244466 (2013). 